\title{
Assembly of Covalently Inorganic-Organic Hybrid Molecular Framework Based on Porphyrin and Phthalocyanine Derivatives-Sensitizer for Dye Sensitized Solar Cells
}

\author{
Hui Lin, Yahong Wu, Shanling Tong, Sheng Hu, Hengde Li, Chuanyi Pan, Yan Yan* \\ College of Chemical Engineering \& Light Industry, Guangdong University of Technology, Guangzhou, China \\ Email: *yanyan600716@hotmail.com
}

How to cite this paper: Lin, $\mathrm{H}$., Wu, Y.H., Tong, S.L., Hu, S., Li, H.D., Pan, C.Y. and Yan, Y. (2018) Assembly of Covalently Inorganic-Organic Hybrid Molecular Framework Based on Porphyrin and Phthalocyanine Derivatives-Sensitizer for Dye Sensitized Solar Cells. Open Journal of Inorganic Chemistry, 8, 1-20.

https://doi.org/10.4236/ojic.2018.81001

Received: October 26, 2017

Accepted: December 10, 2017

Published: December 13, 2017

Copyright $\odot 2018$ by authors and Scientific Research Publishing Inc. This work is licensed under the Creative Commons Attribution International License (CC BY 4.0).

http://creativecommons.org/licenses/by/4.0/

\begin{abstract}
The research on sensitizer for dye sensitized solar cells based on (metallo)porphyrin/phthalocyanine materials were reviewed, and experimental design and assembly method were advised. Latest progress and research status of sensitizer dyes based on metalloporphyrins applied in dye sensitized solar cells was summarized. The preparation and construction of sensitizer electrodes and dye sensitized solar cells based on metal organic frameworks (MOFs) of (metallo)porphyrin/phthalocyanine were projected.
\end{abstract}

\section{Keywords}

Sensitizer, Metalloporphyrin, Phthalocyanine, Frameworks, Dye Sensitized Solar Cells

\section{Introduction}

Functional macrocycle complexes, metalloporphyrins, possess abundant photoelectric properties and molecular assembly model. For example, amidation of porphyrins single amino-group with tricarboxylic acid constructed a special superposed configuration with a helical porphyrin trimmer [1]. A zinc porphyrin dodecamer was assembled successfully, and its electron spectrum was moved towards near-infrared [2]; and a sandwich porphyrin dimmer with a fullerene core was composed a "peasecod" supramolecular complex [3]. Meanwhile, as photosensitive dye, the simple asymmetric zinc porphyrin molecules in dye sen- 
sitized solar cell can increase the photoelectric transformation efficiency with $12 \%-13 \%$ [4] [5]. As functional material, metalloporphyrins were also applied as catalysts in bionic catalysis (i.e., when using manganese porphyrin as catalyst, the $\mathrm{C}-\mathrm{H}$ bond was activated to react with fluorinion to convert organics into homologue fluorides [6].) Conjugate linker of formamido-phenyl porphyrin and $\mathrm{SiC} / \mathrm{SiOx}$ nanowire was exacted by X-ray with photochromic effect to produce singlet oxygen $\left({ }^{1} \mathrm{O}_{2}\right)$, and the singlet oxygen was applied in photodynamic therapy to kill tumor and cancer cells [7]. As molecular wires, porphyrin oligomer with conjugate ethyne linkers performed long distance electron tunnels with low energy consumption [8]. In simulator investigation of cytochrome P450, molecular oxygen can be activated by iron and manganese porphyrin model complexes [9]. Exactly with axial coordination of hexa pyridyl benzene, zinc tetra(4-ethynyl phenyl)porphyrin covalently bonded to construct a macrocyclic oligomer with 12 porphyrin rings, in distorted shape of 8 , even the oligomer shaped as a single circle if a large axial ligand molecule was adopted [10]. All porphyrin rings were covalently linked together with conjugated ethynyl-phenyl bonding (-ph- $\mathrm{C} \equiv \mathrm{C}-\mathrm{C} \equiv \mathrm{C}-\mathrm{ph}-$ ), and every central zinc in porphyrin rings was axially coordinated by the ligating molecule. In supramolecular assembly of porphyrins, notable work was the planar networks based on Schiff base connection covalently, and these porphyrin materials were efficient catalyst for reduction of $\mathrm{CO}_{2}$ in aqueous solution [11].

In this molecular assembly, the aldimine condensation was applied to construct the porphyrin networks, and several same condensations in porphyrin modification were also reported. A series of tetra Shiff base-porphyrins and a dimmer Schiff base porphyrin were successfully prepared [12] [13]. The former revealed a strange electronic effect. The electron donating ability form the secondary substituted phenyls were as below: for the metal free Schiff base porphyrins 1 - 4, p-MeOPhenyl < Phenyl < p-AcOPhenyl < $p$ - $\mathrm{NO}_{2}$ Phenyl; and for zinc-Schiff base porphyrins 5 - 8: Phenyl $<p$-MeOPhenyl $<p$-AcOPhenyl $<$ p- $\mathrm{NO}_{2}$ Phenyl. All second phenyls with substituent were stronger electron donating group than the phenyl itself. The latter was sensitive to recognize inorganic and small organic acids. Metalloporphyrins were efficient catalysts in electrocarboxylations of carbon dioxide with organic halides for getting carboxylic acids with odd carbon atoms, which were rare in nature [14].

A banded structure of whole conjugated metalloporphyrin resulted in extreme decrease of $\pi$ electron transition gap, and its electron spectrum appeared at infrared region with large scale red shift [15]. Remarkably, a modified single metalloporphyrin in dye sensitized solar cell (DSSC) improved photoelectric conversion efficiency in large scale up to $12 \%-13 \%$ [4] [5]. It is expected that metalloporphyrin/phthalocyanine COFs with $\mathrm{D}-\pi-\mathrm{A}$ (electron donor- $\pi$ bond transfer-electron acceptor) configuration would improve the photoelectric effect in DSSC.

In molecular assembly, metalloporphyrins also exhibited their outstanding 
characters. By amidating, mono aminophenyl-trialkoxyphenyl porphyrins and tricarboxylic acids were constructed pretty triple right-handed helices [1] [16]; and by axial coordination of peripheral groups to central metals, tetrahydroxyphenyl porphyrin manganese (III) was assembled as linear right-handed helixes [17]. These supramolecular helixes with optical activity reproduced the configuration of DNA, and its single right-handed construction was natural selectivity. Revealing this profound mystery in helical selectivity would be great research project!

Above discussion indicated that as macrocyclic complexes, metalloporphyrins possessed important research significations and invaluable potential in developing molecular devices for photoelectric conversion and other functional material assembly. Therefore we proposed a research plan to prepare planar networks and steric frameworks based on porphyrins/phthalocyanine complexes, and they would be applied in dye sensitized solar cell (DSSC) to improved photoelectric conversion efficiency.

This research proposal mainly focused on the synthesis of covalently bonded porphyrin/phthalocyanine networks and frameworks and their application in dye sensitized solar cell, and attempted to find out the regularity in hybrid molecular assembly, to reveal their novel functions in photoelectric conversion. By in situ synthesis, porphyrin/phthalocyanine complexes would be directly bonded with bridge molecules to construct hybrid supramolecular material for improving photoelectric conversion efficiency and transmission efficiency, and therefore to develop sensitive detection of photoelectric properties and application of molecular devices, to realize the value for both theoretic research and application on photo/electric/magnetic molecular devices.

In this paper, several reported results on dye sensitized solar cells with macrocyclic sensitizers were reviewed; and the synthetic strategies of novel sensitizers based on metal-organic frameworks (MOfs, or covalent organic frameworks, COFs) of porphyrin/phthalocyanine and their derivatives were proposed based on typical chemical reaction to construct MOFs (COFs); and finally, typically solar cells with these novel MOFs (COFs) as sensitizers were designed to be assembled.

\section{Progress and Research Status of Macrocyclic Complexes Applied in Dye Sensitized Solar Cells}

\subsection{Research Results about Dye Sensitized Solar Cells Based on Macrocyclic Complexes}

Much attention has been paid to metalloporphyrins as chromophores in research area of dye sensitized solar cell (DSSC). In research of inorganic or organic solar cells, graphene-metalloporphyrin was selected as counter electrode material [4] [5]. Physical and chemical assembly and construction based on porphyrin derivatives with graphenes extensively exhibited the broad photoabsorption and photoelectric conversion ability for porphyrin derivatives, and rapid electron transfer function for graphenes. These composite materials with 
excellent photoelectric conversion and energy storage properties were certainly preferred sensitive dyes in dye sensitized solar cells [17]. For enhancing electron transfer effect, in all meso-substituted phenylporphyrin, the substituents were almost selected as carboxyls (-COOH) and ester functions (-COOR) in DSSC research, since the oxygen atoms were easy to bond with conductive substrate to construct efficiently D- $\pi$-A (electron donor- $\pi$ bond transfer-electron acceptor) system. Additionally, carboxyls $(-\mathrm{COOH})$ and ester functions (-COOR) connected well with conductive metal oxides on electrode plates for purpose of rapid electron transfer [18]. For enhance electron transfer efficiency, meso-substituted phenylporphyrins were covalently linked in conjugation way to form an $\mathrm{A}-\pi-\mathrm{D}-\pi-\mathrm{A}$ (electron acceptor- $\pi$ bond transfer-porphyrin electron donor $-\pi$ bond transfer-electron acceptor) system [19]. This photosensitized dye captured light with high performance, and the exited electron can be rapidly transferred by the conjugated system, and therefore it exhibited excellently photovoltaic property. By coordination with zinc ions, meso-tetra (4-carboxyphenyl)porphyrins, and symmetric diamimophenyl porphyrins were constructed a metallic organic frameworks (MOFs), which can efficiently capture photon energy and realize the energy in high-speed transmission [20].

As a linker, trichloro triazine covalently bridge aminophenyl porphyrins together to form porphyrin dimmer and trimmer, which can be used as outstanding photosensitizers in DSSC [21]. Light-harvesting organic metal photosensitizers with "electron donor-acceptor effect" can improve the DSSC's working efficiency. After asymmetric modification on single porphyrin molecule both at peripheral and at central, the complexes with electron donor- $\pi$ bridge-electron acceptor $(D-\pi-A)$ configuration were successfully prepared [22]. Most excellent result about porphyrin sensitizer has been reported belongs to the reported results to improve photoelectric conversion efficiency in scale up to $12 \%-13 \%$ [4] [5].

\subsection{Other Close Research on Molecular Assembly and Applications of Macrocyclic Complexes}

MOFs based on porphyrin-metal complexes can selectively absorb small molecules, such as $\mathrm{CO}_{2}, \mathrm{CO}$, and $\mathrm{CH}_{4}$, et al. [23], to activate these small molecules in catalysis, especially, selectively absorb $\mathrm{CO}_{2}$ [24], and realize its catalytic electroreduction. Film of tetra(4-carboxyphenyl)porphyrin Fe complex-Zr-O cluster possessed high face concentration, and as catalysts for electroreduction from $\mathrm{CO}_{2}$ to $\mathrm{CO}$, the overpotential was efficiently reduced [25]. The similar film of carboxyphenyl porphyrin Co complex-Al-O cluster supplied the same catalytic behavior [26]. Different MOFs based on tetra(4-carboxyphenyl)porphyrin Fe complex-Zr-O cluster can also be selected as catalyst in catalytic conversion from $\mathrm{CO}_{2}$ to ethylene glycol lactone [27], and in oxidative coupling of pyrogallol to produce bicycle compounds by $\mathrm{H}_{2} \mathrm{O}_{2}$ oxidant [28]. Additionally, porphyrin MOFs as hemachrome model compounds can activate molecular oxygen [29]; as catalysts in $\mathrm{O}_{2}$ electroreduction [30] [31]; in oxidation of phenol and sulfoether 
derivatives [32]; and also in water photolysis to produce clear energy source $\mathrm{H}_{2}$ [33]. Another MOFs of tetra(4-carboxyphenyl)porphyrin Sn complex-W-O cluster possessed multifunctional activity in catalysis [34]. Notably, triazine bridged Zn porphyrin's MFOs can recognize and separate small organic molecules [35]. Since porphyrin based MOFs supplied polyfunctionality in different research areas, their molecular assembly and synthesis were paid more research attention. Recently, a specialized commentary about heterogeneous catalysis was reviewed [36].

Assembly types of MOFs based on porphyrin/phthalocyanine were summarized as: 1) porphyrin molecules (peripheral groups were usually selected as carboxyl) were combined with hard acid-O clusters, and the hard acids were high valence ions, including $\mathrm{Al}$ and $\mathrm{Zr}$ [25] [26] [27] [28]; 2) porphyrin molecules were linked with complexes (i.e., Ni-pyridine complexes) [29]; 3) peripheral-central axial coordination and bridge axial coordination constructed crystal porphyrin MOFs; and 4) covalent bond bridged porphyrins mainly depended on covalently conjugate pair of Schiff base bonds, which were constructed by the condensation between aminophenyl porphyrins with $p$-phthalaldehyde and with biphenyl-4, 4'-dicarboxaldehyde. These MOFs were also known as COFs (Covalent Organic Frameworks) [11] [30]-[38].

Porous MOFs of porphyrin/phthalocyanine derivatives can selectively absorb and activate small inorganic and organic molecules; moreover the different properties between central metal and peripheral substituent; the different assembly models, including non-valent and covalent linkage; endow MOFs of porphyrin/phthalocyanine derivatives with abundant photoelectric properties. Therefore these MOFs were always selected as candidates in molecular devices, including photosensitizers in dye sensitized solar cells; sensitive probes in molecule recognitions; and highly active and selective catalysts in catalysis. The most attractive functions were the photoelectric conversion/energy transfer based on MOFs of porphyrin/phthalocyanine derivatives.

Till now seldom research on dye sensitized solar cells using covalent MOFs or COFs, the esterification, amidation and Schiff base condensation products, were reported. Therefore here we designed several porphyrin/phthalocyanine MOFs and COFs, and except exploring their synthesis, assembly, characterization and property determination. The prepared MOFs and COFs would be applied as sensitizer in DSSCs for improving photoelectric transformation efficiency, and they were also used as catalysts in special reaction and molecular recognition. The creative thinking is exhibited in the structure design and molecular device applications.

\section{Primary Coverage}

The reported research indicated that almost all MOFs materials of (metallo)porphyrin/phthalocyanine were adopted the electrovalent coordination of peripheral carboxyl with metal clusters. This electrovalent coordination is not stable enough to sustain the MOFs structures. The stable covalent coordination was 
adopted as Schiff base connection, and several delectable research results were reported. In this project, we would employ (metallo)porphyrins and phthalocyanines with peripherally active groups, including hydroxyls and amino groups, and by covalently linking with bridge moelecules with multi groups of carboxyl, formyl, amino groups and acyl chlorides to construct novel networks and frameworks of porphyrin/phthalocyanine derivatives, and the covalent interaction in the COFs would be ester, amido and Schiff base bonds.

For preparation efficient sensitizers based on metalloporphyrin/phthalocyanine derivatives, covalent bonded COFs with A- $\pi$-D configurations must be designed in synthetic strategy. Except conjugated macrocyclic complexes, all bridge linkers were selected with aromatic structures for enhancing the A- $\pi$-D configurations.

\subsection{Chloridization}

Chlorides of terephthalic acid and benzenetricarboxylic acid were synthesized by using relative acids and thionyl chloride as raw materials. These organic chlorides will directly react with tetra(4-hydroxyphenyl)porphyrin and tetra(4-aminophenyl) porphyrin/phthalocyanines and their complexes to construct the expected 2 dimensional networks and 3 dimensional frameworks (COFs). The bridge molecules would be selected as multi organic acids, 2, 4, 6-trichloro-[1, 3, 5]-triazine; phthalaldehyde, isophthalaldehyde, orthophthalaldehyde, and biphenyl-4, 4'-dicarbaldehyde, et al.

\subsection{Controlling Sizes of Channels and Cavities in COFs}

The sizes of channels and cavities in the COFs were controlled by selecting different size of bridge molecules for convenient recognizing and activating small molecules in catalytic conversion of $\mathrm{CO}_{2}, \mathrm{H}_{2} \mathrm{O}, \mathrm{H}_{2} \mathrm{O}_{2}, \mathrm{CO}, \mathrm{CH}_{4}, \mathrm{CH}_{3} \mathrm{CHO}$ and $\mathrm{CH}_{2} \mathrm{O}$.

\subsection{Electronic Configuration of the COFs}

The intended COFs of (metallo)porphyrin/phthalocyanine derivatives integrate electron donor-electron acceptor (D-A), the integrated D-A model omit the segment of $\pi$ electron transfer, and realize directly photoelectron transporting from donor to acceptor, and therefore photoelectric effect of the MOFs will be much enhanced.

\subsection{COFs Electrodes}

Electrode of the intended COFs of (metallo)porphyrin/phthalocyanine derivatives would be prepared and their functionality would be explored, such as hydrogen production in photochemical water splitting for getting clean energy source; electrocatalysis of $\mathrm{CO}_{2}$ for small organic compound preparation; $4 \mathrm{e}^{-}$reduction of oxygen molecules for increasing atomic economics; and related molecular recognition for selective separation of mixture gases. 


\subsection{Design of DSSCs with the COFs}

Preparation of dye sensitized solar cells with sensitizer of the intended COFs of (metallo)porphyrin/phthalocyanine derivatives would supplied novel energy resources for protecting environment, realizing energy-saving and emission-reduction. Therefore this proposal is a completely new attempt.

\section{Research Program}

\subsection{Synthesis of Porphyrin/Phthalocyanine with Tetra(Hydroxyphenyl) and Tetra(Aminophenyl) and Their Complexes}

The porphyrins, phthalocyanine and their complexes for assembly of the intended MOFs or COFs were described in Scheme 1. Porphyrins and related complexes were prepared according to the reported references (a. J. Inorg. Nucl. Chem., 1970, 32, 2443; b. J. Org. Chem., 1987, 52, 827.). Synthetic method of amino phthalocyanine and its complexes was summarized from our experimental exploring.

\subsection{Covalently Planar Networks and Stereo Frameworks of (Metallo)Porphyrin/Phthalocyanine Derivatives}

Terephthalic acid, isophthalic acid and benzenetricarboxylic acid react with thionyl chloride to obtain their relative acylchlorides. After esterification or
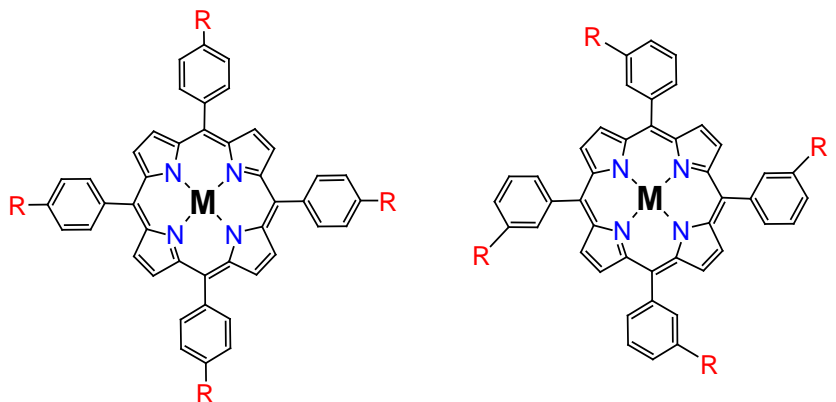

$$
\begin{aligned}
& \mathrm{R}=-\mathrm{OH},-\mathrm{NH}_{2} \\
& \mathrm{M}=2 \mathrm{H}^{+}, \mathrm{Zn}^{\prime \prime}, \mathrm{Fe}^{\mathrm{III/I}}, \mathrm{Co}^{\prime \prime}, \mathrm{Ni}^{\mathrm{II}}, \mathrm{Mn}^{\mathrm{III/I}}, \mathrm{Cu}^{\prime \prime}, \mathrm{Cd}^{\prime \prime}, \mathrm{Ti}^{\mathrm{IV} / \| I}, \mathrm{Sn}^{\mathrm{IV}} \ldots . .
\end{aligned}
$$

(a)

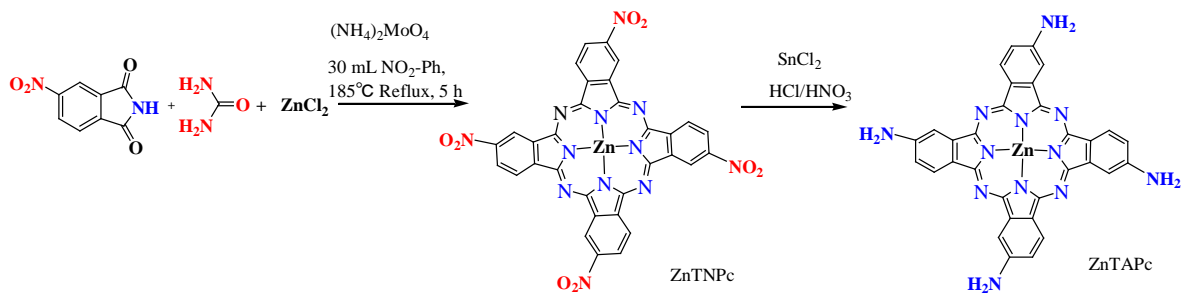

(b)

Scheme 1. Molecule structures and synthesis of the (metallo)porphyrins, phthalocyanine and their related complexes for constructing the intended COFs (All molecules were designed as symmetric or asymmetric ones for increasing formation probability of ester and amido bonds). 
amidation, these acyl chlorides and tetra(hydroxyphenyl)-, tetra(aminophenyl)porphyrins and tetra(aminophenyl)-phthalocyanine would be constructed into the intended COFs. (Figure 1) Experimental indicated that direct esterification and amidation were incomplete reactions. For activating, all bridged organic acids were converted in to their relative acyl chlorides for constructing the covalent bonding interaction. If the bridge molecules were selected as multiple phenylaldehydes, multiple chlorobenzene, and even trichloro triazine, the COFs would be directly constructed without bridged molecule activation. The bridged molecule structures were given in Figure 2.

\subsection{Characterization and Properties Determination of (Metallo)Porphyrin/Phthalocyanine COFs}

Properties and crystal morphology of the novel (metallo)porphyrin/phthalocyanine COFs would be characterized and determined by spectral and electron microscopic analysis. Especially, the functions of photocatalysis and molecular recognitions of the novel COFs would be explored and investigated intensively.
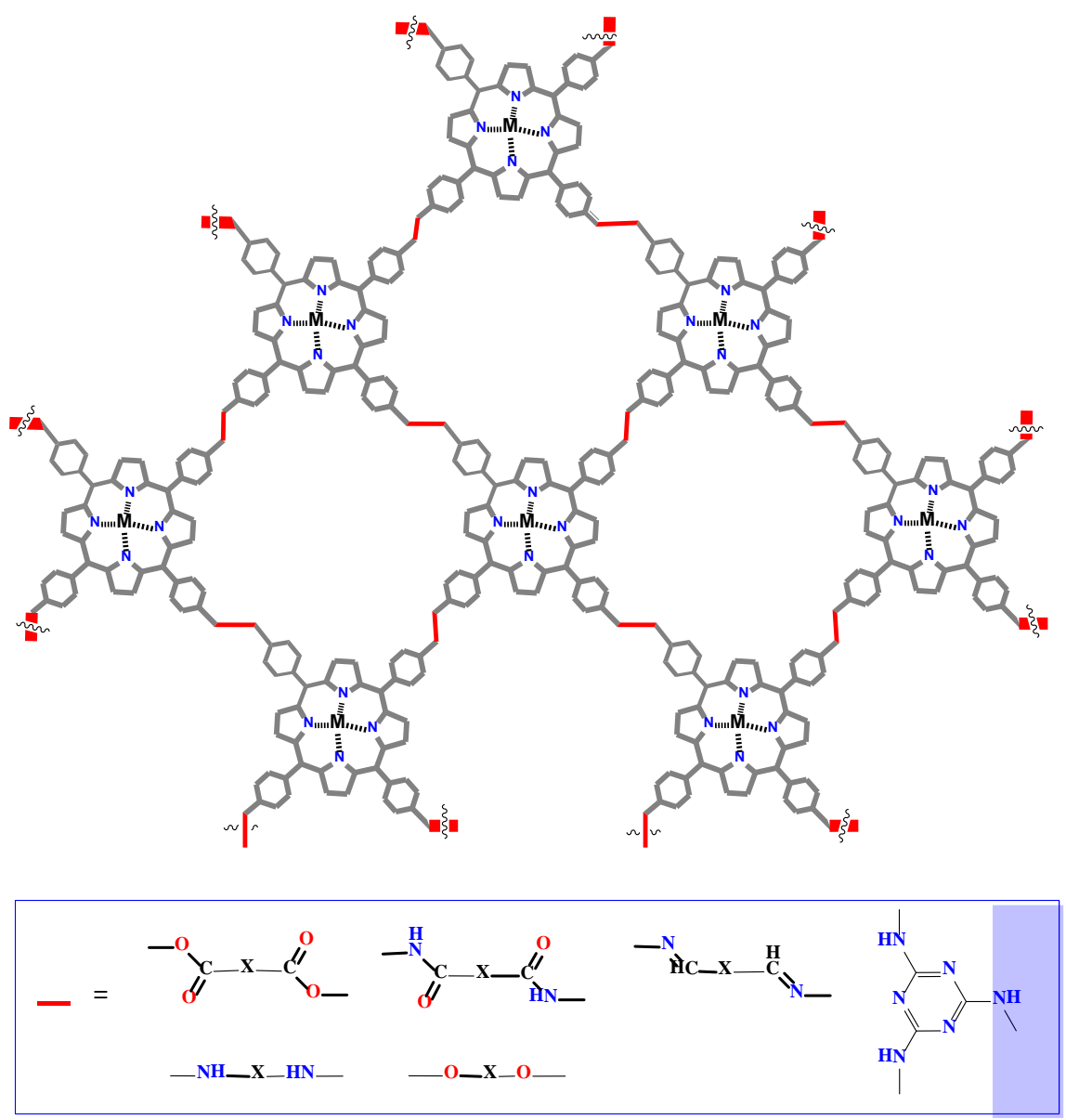

Figure 1. Planar networks of (metallo)porphyrin derivatives (COFs). X represented the skeleton in bridge molecules (Diagrams of 3D frameworks and phthalocyanine related were omitted for clarity). 

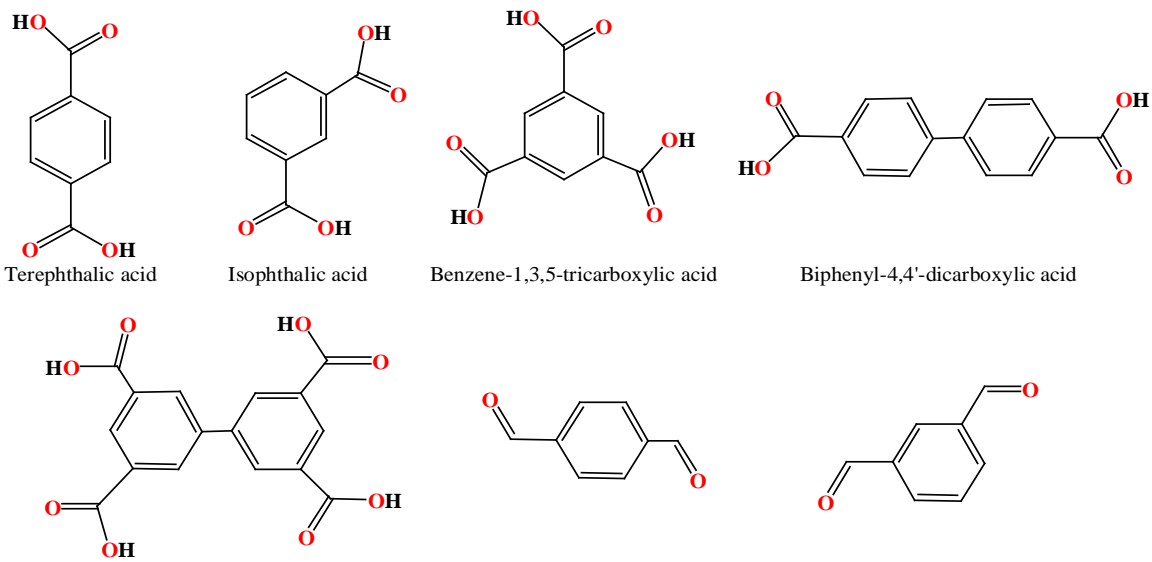

Benzene-1,3,5-tricarboxylic acid

Biphenyl-4,4'-dicarboxylic acid
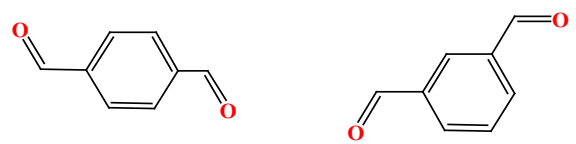

Biphenyl-3,5,3',5'-tetracarboxylic acid

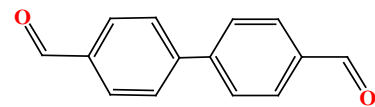

Benzene-1,4-dicarbaldehyde
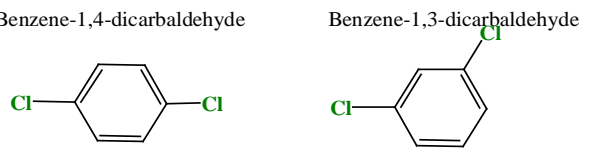

Biphenyl-4,4'-dicarbaldehyde

1,4-Dichloro-benzene

1,3-Dichloro-benzene
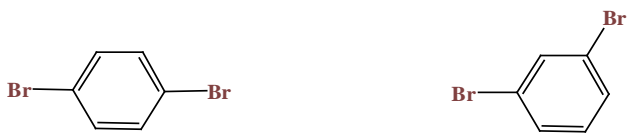<smiles>Clc1nc(Cl)nc(Cl)n1</smiles>

1,4-Dibromo-benzene

1,3-Dibromo-benzene

2,4,6-Trichloro-[1,3,5]triazine

Figure 2. Candidates selected as bridge molecules in construction of porphyrin/phthalocyanine COFs.

\subsection{Photoelectric Transition Efficiencies}

The COFs of porphyrin/phthalocyanine derivatives would be assembled as photo permeable electrodes and their photovoltaic effects and photocurrent intensity would be determined. These electrodes would be used in electrocatalysis and photoelectrocatalysis. Finally, novel dye sensitized solar cells would be designed and assembled with this photo permeable electrodes, and the cells efficiencies would be examined and improved. According to reported method [19], the designed DSSCs' diagram was showed in Figure 3.

\subsection{Key Steps in Research}

1) Assemble conditions in assembly of COFs of porphyrin/phthalocyanine derivatives would be optimized, including selections of central metals, peripheral groups, bridge molecules and their multi function groups, reaction types for covalent bond formation. Exploring bridging reaction conditions for constructing COFs of (metallo)porphyrin/phthalocyanine derivatives with excellent photoelectric effects; 2) The intended COFs of (metallo)porphyrin/phthalocyanine derivatives would be designed and constructed as electrodes and even the intended DSSC, and the properties of these molecular devices would be evaluated by extensive determinations, including facial load, DSSC preparation and functional examination. In situ determination would be also employed by combine determinations of photoelectrochemistry, photoelectro-EPR monitoring, designation 


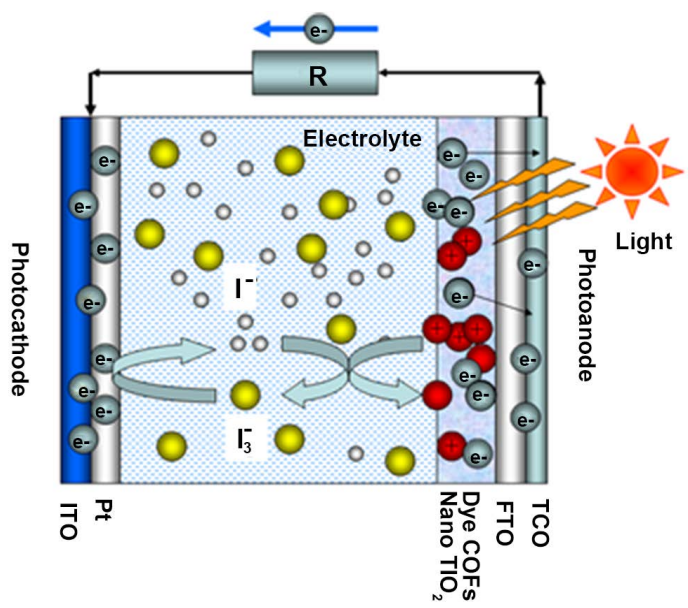

Figure 3. Diagram of the intended DSSC with Dye COFs of porphyrin/phthalocyanine derivatives. TCO = Transparent conductive oxide; FTO $=$ F-doped tinoxide; ITO = Indium tin oxide.

and test for photocurrent and photovoltage effects; 3) More attention should be focused on high performance DSSC designation, assembly and their efficiency determinations.

\subsection{Bridging Method}

The thionyl chloride would be employed in chlorination of bridge polyacids for getting more active bridge molecules in constructing COFs of (metallo)porphyrin/phthalocyanine derivatives by esterification and amidation. Other bridging molecules, including di- and tri-halogenated aromatics and aromatic polyaldehydes, could be directly used in etherification and aldimine condensation to construct the purpose COFs. The possible bridging reactions were summarized in Schemes 2-5.

Hydroxyphenyl, aminophenyl porphyrins, and amino phthalocyanine derivatives would be linked together to construct their COFs by esterification and amidation (Scheme 2).

Their COFS also would be constructed by etherification and amino substitution. In this type construction, the bridging molecules would be selected as di- and tri-halogenated aromatics (Scheme 3). Aldol condensation would be employed for constructing the COFs of (metallo)porphyrin/phthalocyanine derivatives resulted Schiff base bond linkage (Scheme 4). Tricloro-triazene would be employed as bridging molecule, too (Scheme 5 ).

Although synthesis of (metallo)porphyrin/phthalocyanine and their derivatives was already mature, the bridging reaction conditions should be explored and improved intensively. For example, in the heterogeneous reactions to construct COFs, phase transfer catalysts, quaternary ammonium salts, would be used to accelerate formation of covalent linkage. In consideration of electron effects and steric effects, the substituents of benzaldehydes and central metals in 


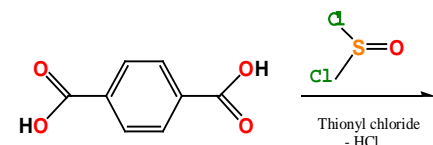<smiles>O=C(Cl)c1ccc(C(=O)Cl)cc1</smiles>

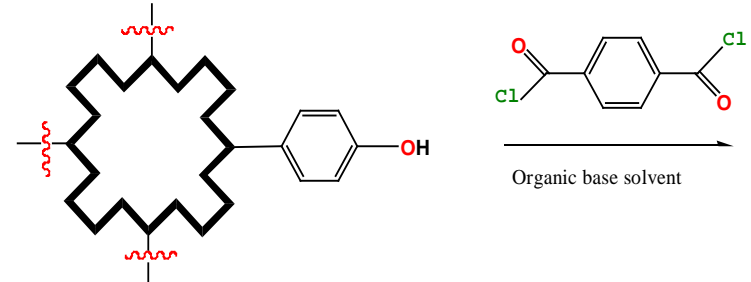
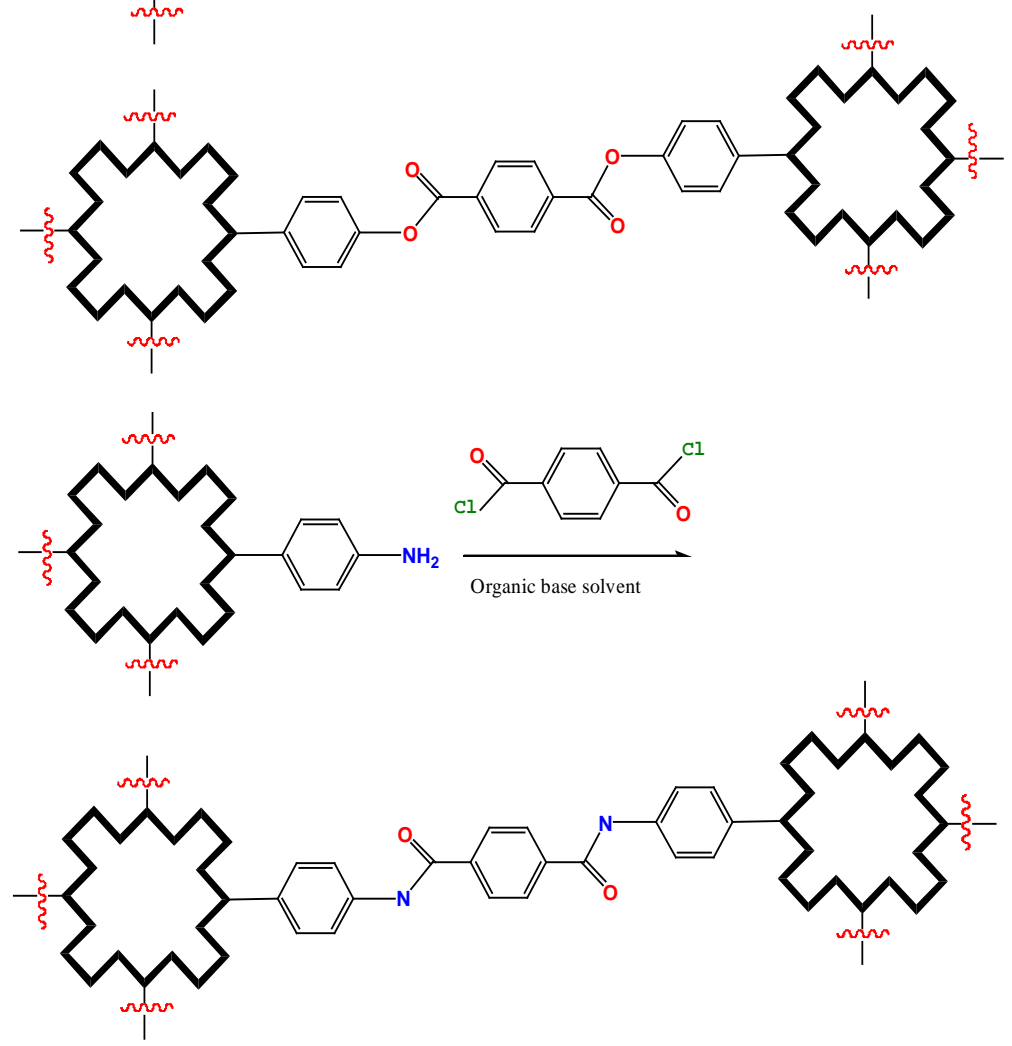

(a)

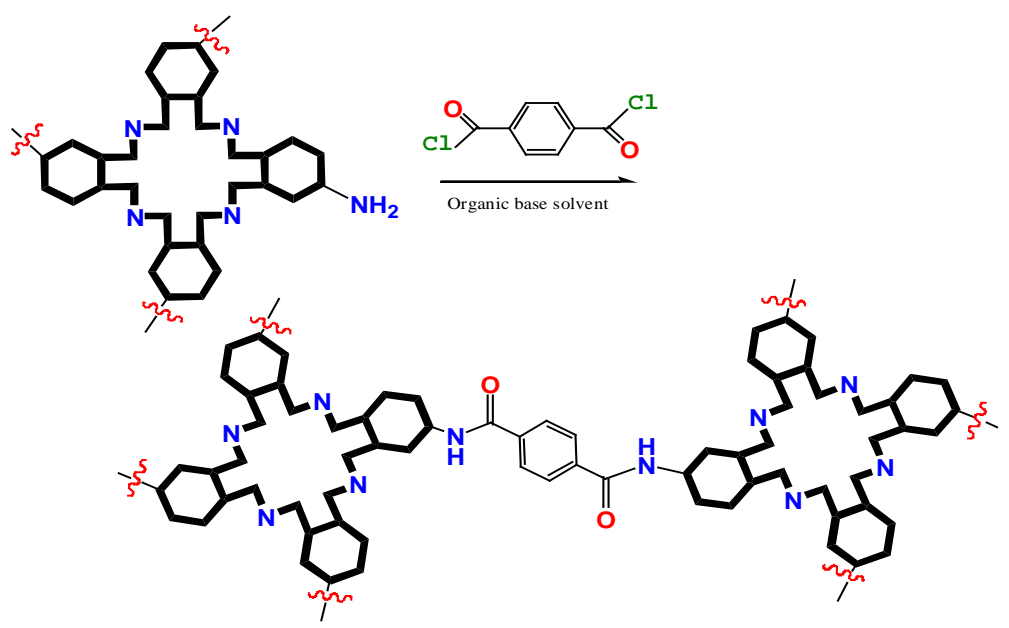

(b)

Scheme 2. Bridging bond formation with esterification and amidation. 

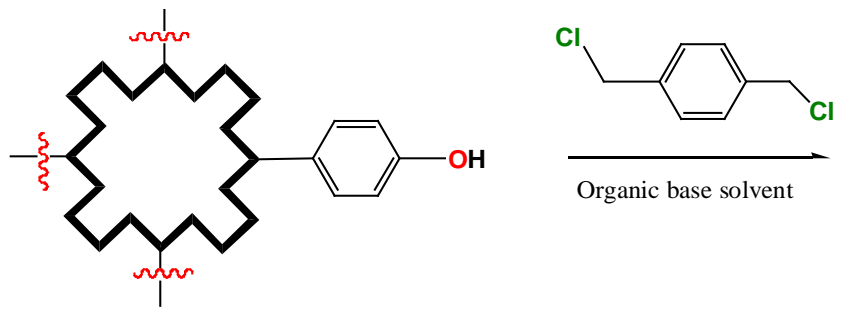

Organic base solvent
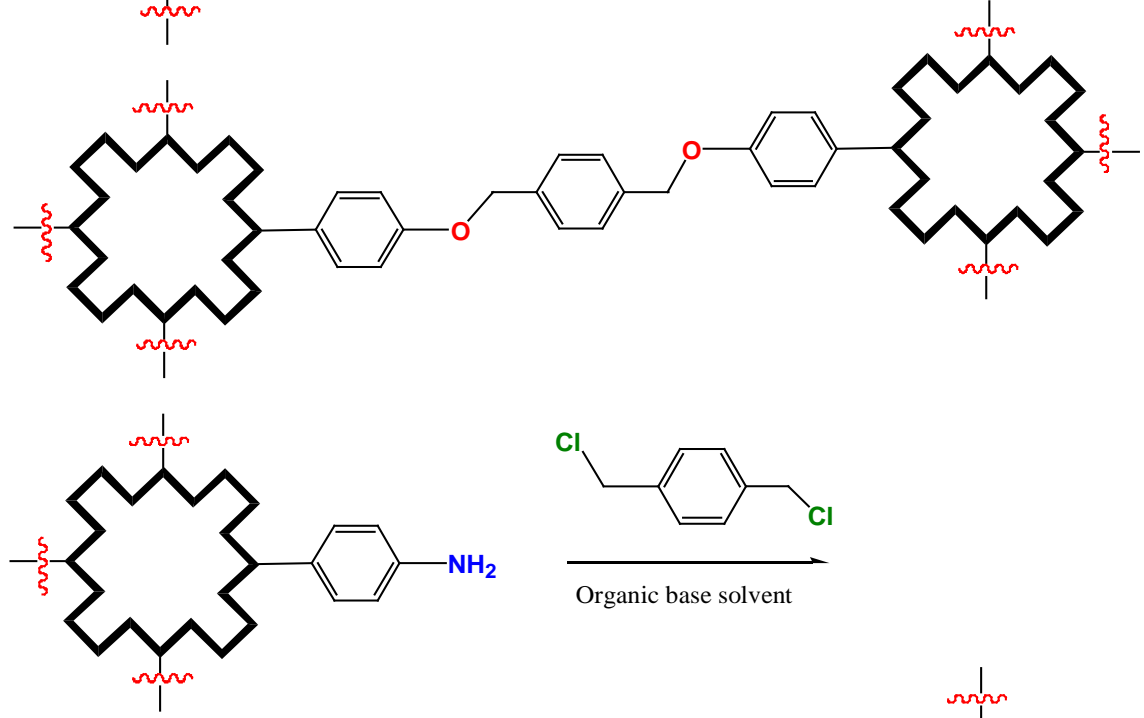

Organic base solvent

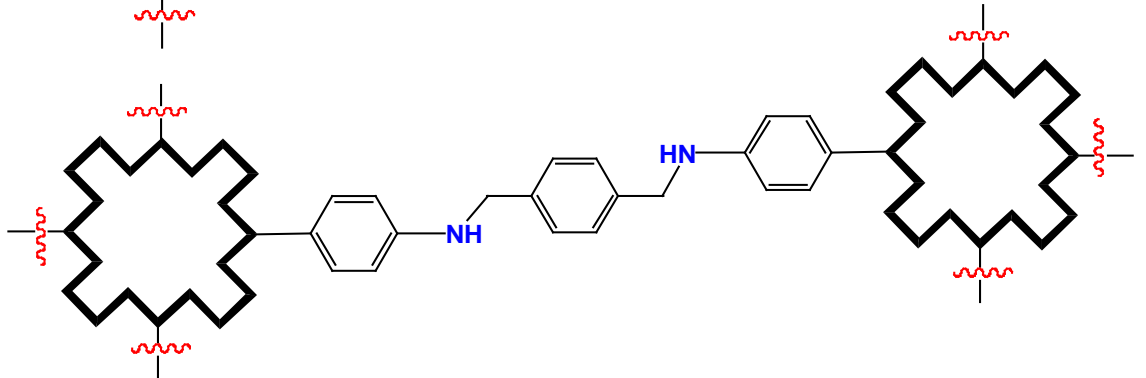

(a)
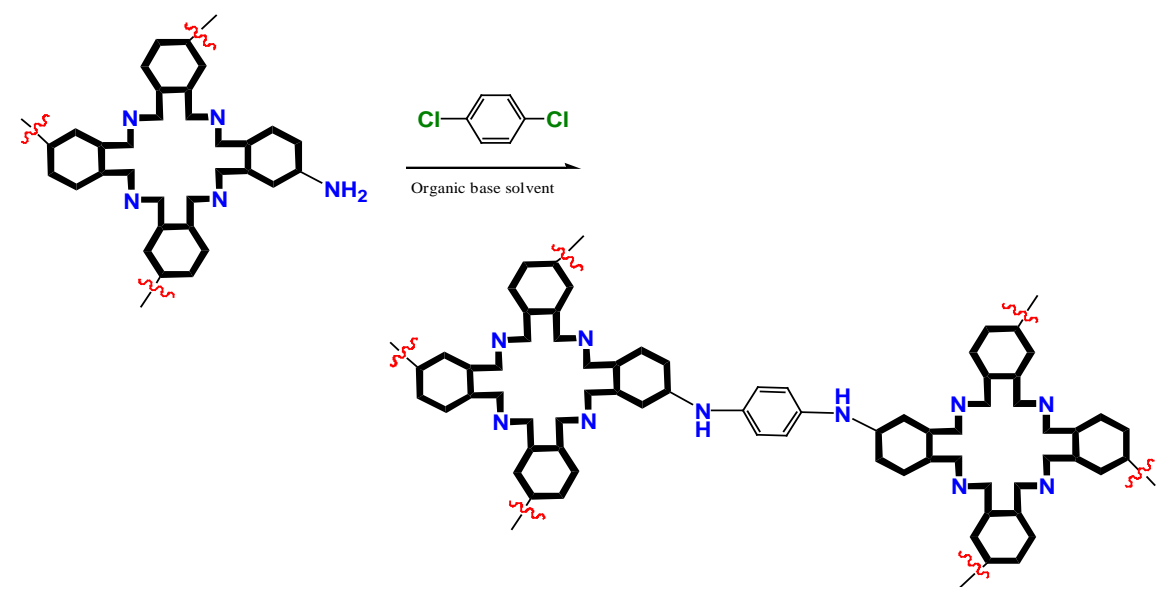

(b)

Scheme 3. COFs constructed by di- and tri-halogenated aromatics.

macrocyclic complexes should be selected, modified and replaced respectively. Beside of covalent bridging, the axial coordination is also a driving force for MPor/MPC COFs construction. 


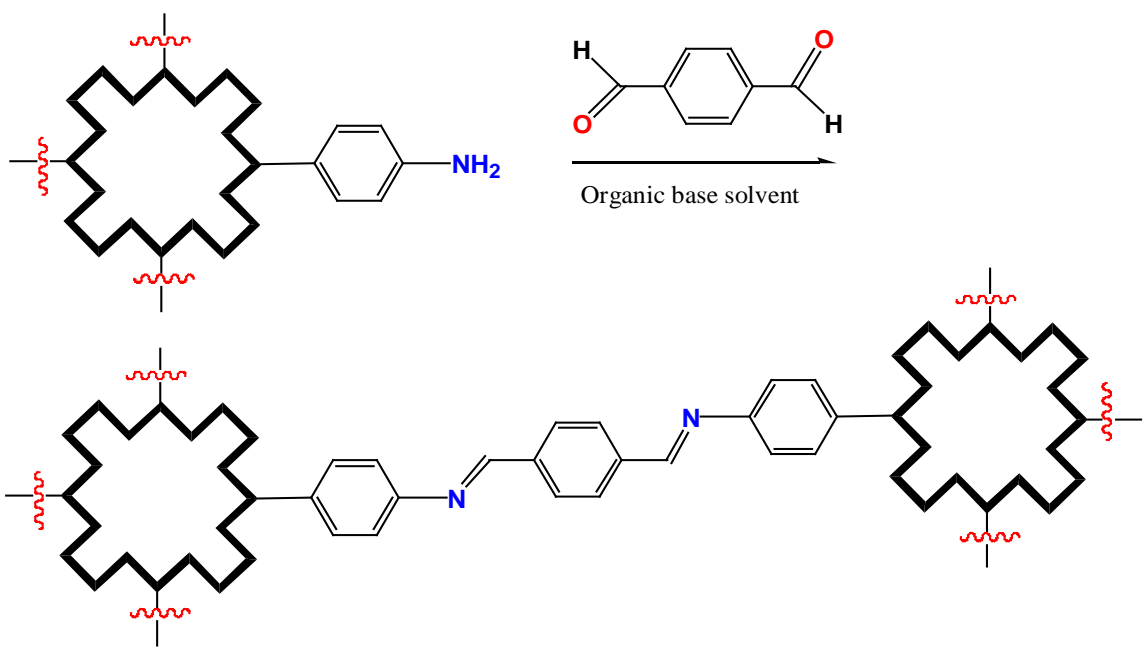

(a)

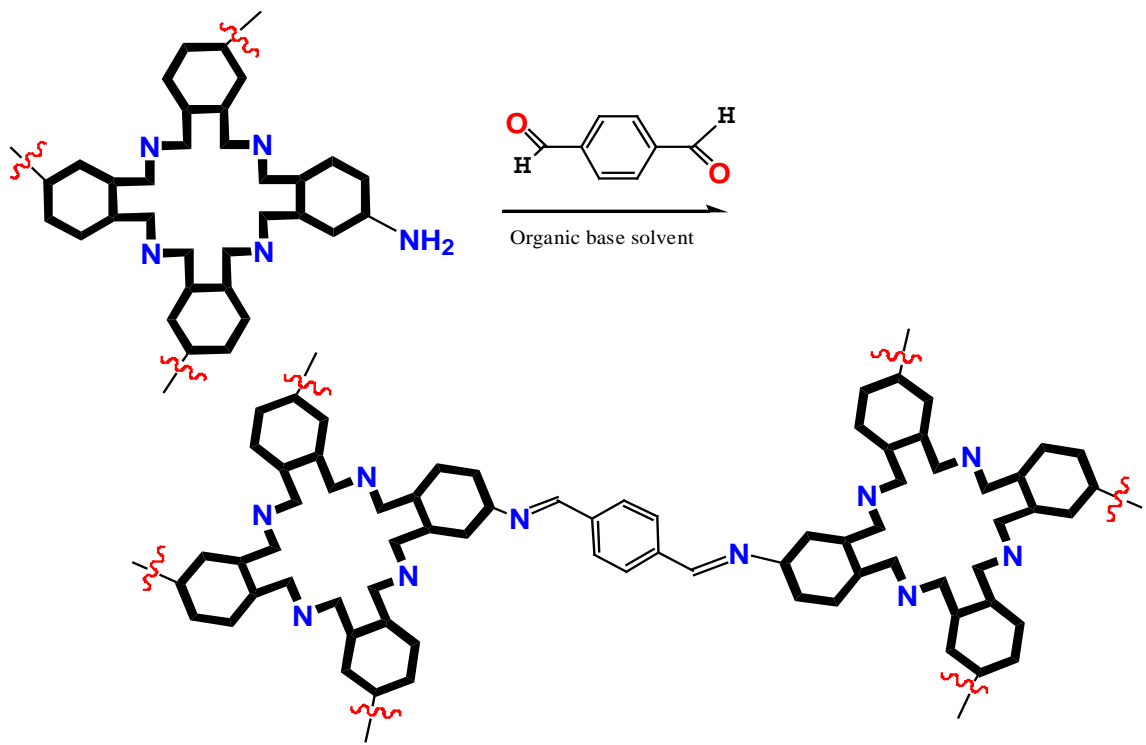

(b)

Scheme 4. Bridging bond formation with aldol condensation.

The sizes and molecular weights of the intended COFs based on (metallo) porphyrin/phthalocyanine derivatives should be efficiently controlled, and moreover with peripheral modification, trying to enhance solubility of the intended COFs in organic and aqueous solutions in convenience for construction of molecular devices.

During synthesis and molecular assembly, instrument and determination method are indispensible. The characterization and property determination would be carried out by employ of UV-Vis, IR, Raman, NMR, EPR, AFM and SEM method.

In electrode preparation, controlling hydrolysis of $\mathrm{Ti}\left(\mathrm{OC}_{2} \mathrm{H}_{5}\right)_{4}$ on the surface of $\mathrm{TCO}$ (Transparent conducting oxide) glasses, and after cementation the $\mathrm{TiO}_{2}$ nanofilm would formed for increasing photoabsorption and physical-chemistry 

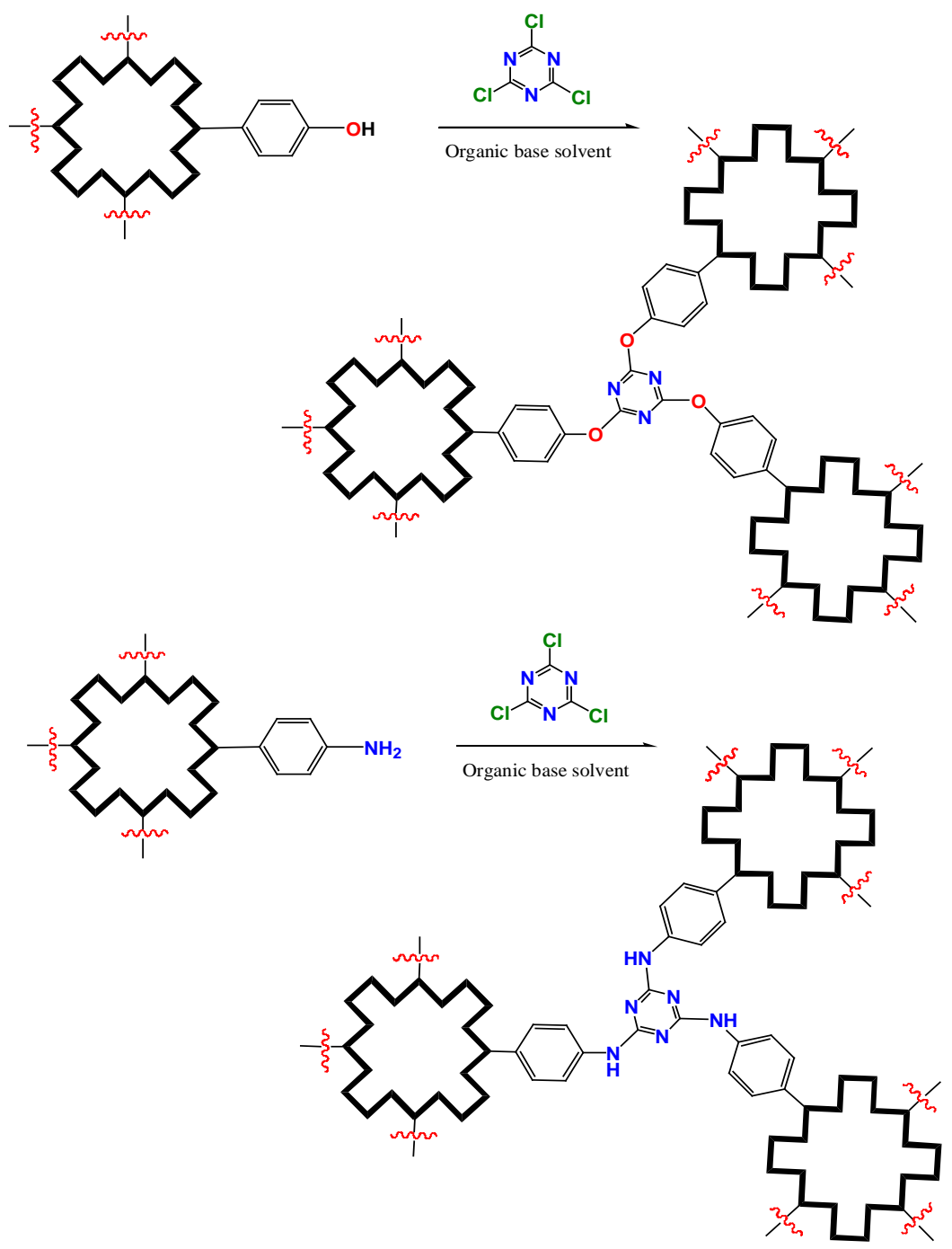

(a)

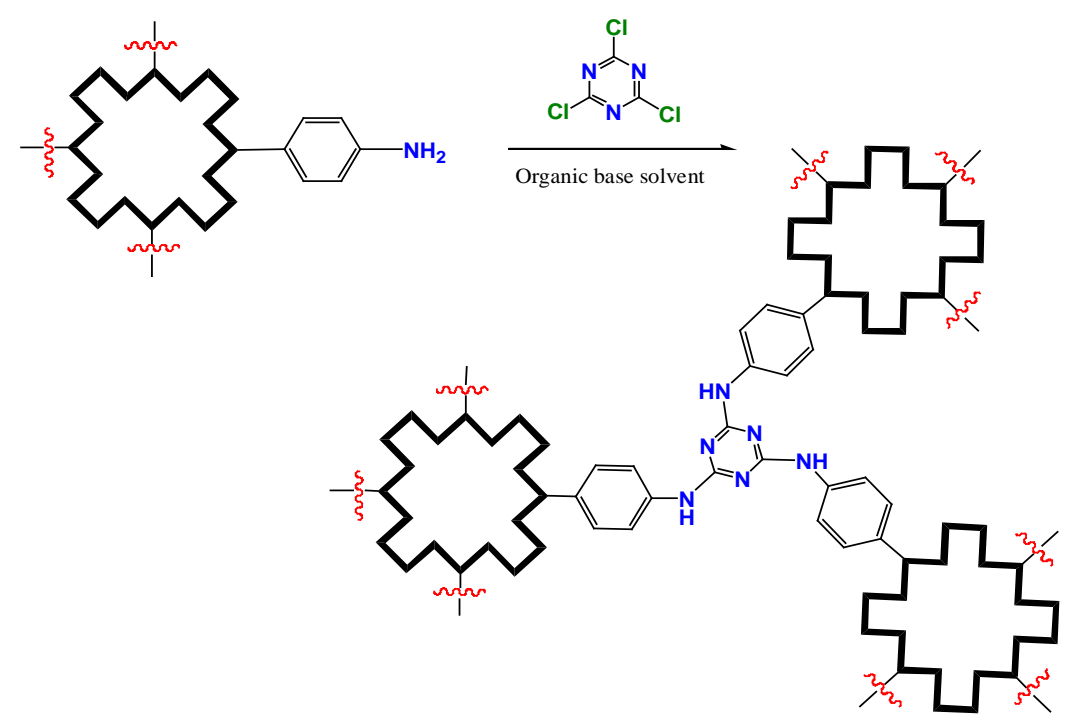

(b)

Scheme 5. COFs constructed by trichloro-triazene. 
adhesion towards the intended COFs. For avoiding COFs' dissolution, these electrodes would be used in solutions with more different polarity in photoelectrochemistry determinations (cyclic voltammetry, constant potential electrolysis, and determinations of photocurrent and photovoltage etc.). Finally, the photo-active electrodes based on the MOFs of (metallo)porphyrin/phthalocyanine derivatives would be efficiently assembled in dye sensitized solar cells for getting novel energy sources.

\subsection{Research Route}

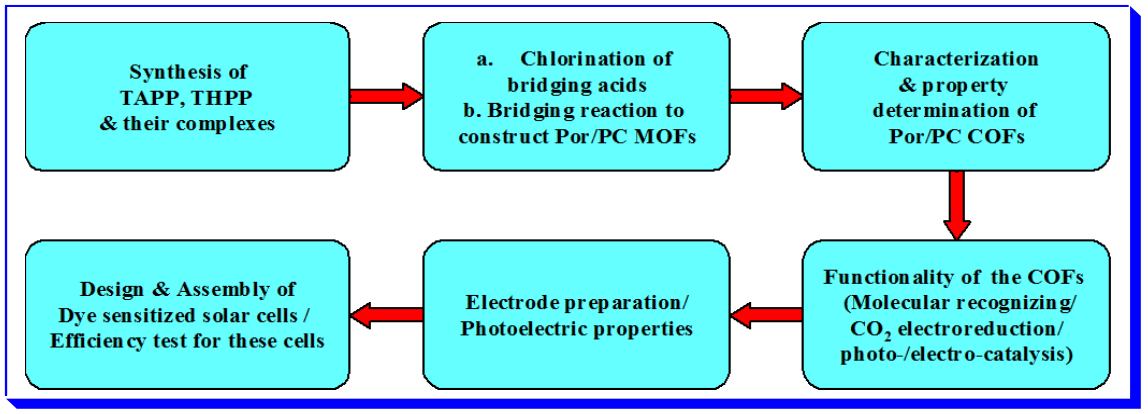

\subsection{Experimental Method}

For activating esterification and amidation, poly acid bridge molecules must be chloridized by thionyl chloride; other selected bridge molecules can be directly used in constructing of MPor/MPC COFs, including networks and stereo frameworks.

During assembly of MPor/MPC COFs, the conditions of inhomogeneous reactions should be paid more attention, and even phase transfer catalyst of quaternary ammonium salts would be employed necessarily. Meanwhile, the polarity of solvents would be selected suitably to accelerate formation of the COFs; and the attention should be paid to the structural characterization and performance testing of the novel materials.

In functional determination experiments, electrochemistry/photochemistry/ spectrochemistry method would be adopted to detect redox, photoelectric conversion and electrolysis properties of the COFs. Some special determination devices, including light permeable thin layer electrochemical cells and EPR-electrochemical cells for in situ determinations, should be designed and constructed.

For preparation of the MPor/MPC COFs electrodes, firstly $\mathrm{Ti}\left(\mathrm{OC}_{2} \mathrm{H}_{5}\right)_{4}$ would be hydrolyzed on the surfaces of ITO glasses (indium tin oxide, light permeable-conductive glasses) under controlling conditions, and followed by heating for completely hydrolysis to convert into $\mathrm{TiO}_{2}$ nanoparticles. Then MPor/MPC COFs would be dispersed in suitable solvents and dipped in the previous treated surfaces of the electrodes. By physical and weak chemical interactions, MPor/MPC COFs would be tightly combined with $\mathrm{TiO}_{2}$ nanoparticles, and these MPor/MPC-COFs- $\mathrm{TiO}_{2}$-ITO electrodes would be applied in photoelectrochem- 
ical catalysis and molecular recognizing to investigate catalytic mechanism molecular recognition efficiencies. Furthermore the electrodes would be assembled the desired dye sensitized solar cells for examinations of their photoelectric conversion efficiency, cell performance and cell life. A highly efficient and energy saving dye sensitized solar cell is expected to be selected for practice application.

\subsection{Key Technology in Research}

a) In situ determination devices, including spectroscopic electrochemistry and EPR-electrochemistry, are needed to be designed and prepared; determination method and relative kinetic research should be developed.

b) Photoelectric conversion efficiency and its influence factors should be confirmed. The relationship between material structure and cell performance should be definitely formalized, and the influence regulation of structure on properties would be summarized.

\subsection{Feasibility Analysis}

Thionyl chloride (dichloro sulfoxide, DCSO) is an efficient reagent for chloridization of organic acid. After chloridization, poly acid chlorides become more active in bridging reaction with hydroxyl- and amino-porphyrin/phthalocyanine derivatives to construct their COFs. Conditions for other bridging reactions, including etherification and imidization with halogenated bridging molecules, would be intensively explored. For realizing complete condensation, the hydroxyl in peripheral hydroxyphenyl around macrocycles should be conversed into phenolic sodium salt to react with halogenated bridging molecules to construct their COFs. In consideration of stereo effect, para-substituted porphyrin derivatives are suitable in assembly of planar networks; while the meta- and ortho-substituted ones are selected as candidates for constructing 3D frameworks.

The intended COFs of Por/PC derivatives would be characterized by normal determinations. Their functional investigation would be focused on efficiency of photocatalysis and biomolecular recognition, including photocatalytic degradation of water and electroreduction of oxygen and carbon dioxide molecules under photoelectric conversion. As results, clean energy sources would be created, the atomic economics of oxygen molecules would be adequately realized, and pollution from $\mathrm{CO}_{2}$ greenhouse gas would be restrained effectively. Our previous research on electrocarboxylation of $\mathrm{CO}_{2}$ [39] accumulated definite experience for this research project.

Preparation of electrodes and DSSC assembly based on the intended COFs of Por/PC derivatives, and the in situ determinations of their properties were all derived from the reliable sources. The research plan would be regarded as the foundation of follow-up application, and be expected to get a breakthrough research results.

The previous research was predominantly concentrated in chemical synthesis and characterizations for macrocyclic compounds. Although applications in photoelectrochemical determination method was established and catalysis in 
$\mathrm{CO}_{2}$ electric reduction [39] and natural product [40] [41] obtained preliminary results, the research was much more different form the applications. Therefore in this project, the research will be focused on molecular device assembly and practice applications of the intended COFs of Por/PC derivatives.

\section{Acknowledgements}

This work was supported by the Natural Science Foundation of Guangdong province (Grant No. 2017A030313071), Excellent Young Teacher Development Project of Universities in Guangdong Province (Grant No. 261532106) and the Guangzhou Science and Technology Program (Grant No. 502150105).

\section{References}

[1] Hameren, R.V., Schön, P., Buul, A.M.V., Hoogboom, J., Lazarenko, S.V., Gerritsen, J.W., Engelkamp, H., Christianen, P.C.M., Heus, H.A., Maan, J.C., Rasing, T., Speller, S., Rowan, A.E., Elemans, J.A.A.W. and Nolte, R.J.M. (2006) Macroscopic Hierarchical Surface Patterning of Porphyrin Trimers via Self-Assembly and Dewetting. Science, 314, 1433-1436. https://doi.org/10.1126/science.1133004

[2] Tsuda, A. and Osuka, A. (2001) Fully Conjugated Porphyrin Tapes with Electronic Absorption Bands That Reach into Infrared. Science, 293, 79-82. https://doi.org/10.1126/science.1059552

[3] Yamaguchi, T., Ishii, N., Tashiro, K. and Aida, T. (2003) Supramolecular Peapods Composed of a Metalloporphyrin Nanotube and Fullerenes. Journal of the American Chemical Society, 125, 13934-13935. https://doi.org/10.1021/ja038178j

[4] Yella, A., Lee, H.-W., Tsao, H.N., Yi, C., Chandiran, A.K., Nazeeruddin, Md.K., Diau, E.W.-G., Yeh, C.-Y., Zakeeruddin, S.M. and Grätzel, M. (2011) Porphyrin-Sensitized Solar Cells with Cobalt (II/III)-Based Redox Electrolyte Exceed 12 Percent Efficiency. Science, 334, 629-634. https://doi.org/10.1126/science.1209688

[5] Mathew, S., Yella, A., Gao, P., Humphry-Baker, R., Curchod, B.F.E., Ashari-Astani, N., Tavernelli, I., Rothlisberger, U., Nazeeruddin, Md.K. and Grätzel, M. (2014) Dye-Sensitized Solar Cells with 13\% Efficiency Achieved through the Molecular Engineering of Porphyrin Sensitizers. Nature Chemistry, 6, 242-247. https://doi.org/10.1038/nchem.1861

[6] Liu, W., Huang, X., Cheng, M.-J., Nielsen, R.J., Goddard III, W.A. and Groves, J.T. (2012) Oxidative Aliphatic C-H Fluorination with Fluoride Ion Catalyzed by a Manganese Porphyrin. Science, 337, 1322-1325.

https://doi.org/10.1126/science.1222327

[7] Rossi, F., Bedogni, E., Bigi, F., Rimoldi, T., Cristofolini, L., Pinelli, S., Alinovi, R., Negri, M., Dhanabalan, S.C., Attolini, G., Fabbri, F., Goldoni, M., Mutti, A., Benecchi, G., Ghetti, C., Iannotta, S. and Salviati, G. (2015) Porphyrin Conjugated $\mathrm{SiC} / \mathrm{SiOx}$ Nanowires for X-Ray-Excited Photodynamic Therapy. Scientific Reports, 5, 7606. https://doi.org/10.1038/srep07606

[8] Sedghi, G., García-Suárez, V.M., Esdaile, L.J., Anderson, H.L., Lambert, C.J., Martín, S., Bethell, D., Higgins, S.J., Elliott, M., Bennett, N., Macdonald, J.E. and Nichols, R.J. (2011) Long-Range Electron Tunnelling in Oligo-Porphyrin Molecular Wires. Nature Nanotechnology, 6517-6523. https://doi.org/10.1038/nnano.2011.111

[9] Guo, M., Dong, H., Li, J., Cheng, B., Huang, Y.-Q., Feng, Y.-Q. and Lei, A. (2012) Spectroscopic Observation of Iodosylarene Metalloporphyrin Adducts and Manganese(V)-Oxo Porphyrin Species in a Cytochrome P450 Analogue. Nature Communi- 
cations, 3, 1190. https://doi.org/10.1038/ncomms2196

[10] O’Sullivan, M.C., Sprafke, J.K., Kondratuk, D.V., Rinfray, C., Claridge, T.D.W., Saywell, A., Blunt, M.O., O’Shea, J.N., Beton, P.H., Malfois, M. and Anderson, H.L. (2011) Vernier Templating and Synthesis of a 12-Porphyrin Nano-Ring. Nature, 469, 72-75. https://doi.org/10.1038/nature09683

[11] Lin, S., Diercks, C.S., Zhang, Y.-B., Kornienko, N., Nichols, E.M., Zhao, Y., Paris, A.R., Kim, D., Yang, P., Yaghi, O.M. and Chang, C.J. (2015) Covalent Organic Frameworks Comprising Cobalt Porphyrins for Catalytic CO2 Reduction in Water. Science, 349, 1208-1213. https://doi.org/10.1126/science.aac8343

[12] Wu, Y., Chen, L., Yu, J., Tong, S. and Yan, Y. (2013) Synthesis and Spectroscopic Characterization of Meso-Tetra (Schiff-Base Substituted Phenyl) Porphyrins and Their Zinc Complexes. Dyes and Pigments, 97, 423-428. https://doi.org/10.1016/j.dyepig.2012.12.032

[13] Wu, Y.H., et al. (2014) Schiff Base Bridged Biporphyrin: Synthesis, Characterization and Spectral Properties. Inorganic Chemistry Communications, 45, 10-14. https://doi.org/10.1016/j.inoche.2014.03.017

[14] Zheng, G.D. (1996) The Reaction Mechanism of Alkyl Halides with Carbon Dioxide Catalyzed by $5,10,15,20$-Tetraphenyl Porphyrin Cobalt (CoTPP). Electrochimica Acta, 41, 177-182. https://doi.org/10.1016/0013-4686(95)00279-N

[15] Tsuda, A. and Osuka, A. (2001) Fully Conjugated Porphyrin Tapes with Electronic Absorption Bands That Reach into Infrared. Scince, 293, 79-82. https://doi.org/10.1126/science.1059552

[16] Yang, J.-W., Zhang, J., Yan, Y., Huang, X.-C. and Tong, S.-L. (2010) A Coordination Polymer of Manganese (III) Porphyrinate with Right-Handed Helicity and Opticity. Solid State Sciences, 12, 1242-1247. https://doi.org/10.1016/j.solidstatesciences.2010.03.003

[17] Wang, S., Liu, X.Y. and Gu, Y.Z. (2016) Excellent Photoelectric Properties and Charge Dynamics of Two Types of Bulk Heterojunction Solar Cells. Materials Letters, 166, 251-254. https://doi.org/10.1016/j.matlet.2015.12.085

[18] Urbani, M., Grätzel, M., Nazeeruddin, M.K. and Torres, T. (2014) Meso-Substituted Porphyrins for Dye-Sensitized Solar Cells. Chemical Reviews, 114, 12330-12396.

[19] Kumar, C.V., Cabau, L., Koukaras, E.N., Sharma, A., Sharma, G.D. and Palomares, A.E. (2015) $-\pi-D-\pi$-A Based Porphyrin for Solution Processed Small Molecule Bulk Heterojunction Solar Cells. Journal of Materials Chemistry A, 3, 16287-16301. https://doi.org/10.1039/C5TA03463A

[20] Son, H.-J., Jin, S., Patwardhan, S., Wezenberg, S.J., Jeong, N.C., So, M., Wilmer, C.E., Sarjeant, A.A., Schatz, G.C., Snurr, R.Q., Farha, O.K., Wiederrecht, G.P. and Hupp, J.T. (2013) Light-Harvesting and Ultrafast Energy Migration in Porphyrin-Based Metal-Organic Frameworks. Journal of the American Chemical Society, 135, 862-869. https://doi.org/10.1021/ja310596a

[21] Zervaki, G.E., Tsaka, V., Vatikioti, A., Georgakaki, I., Nikolaou, V., Sharma, G.D. and Coutsolelos, A.G. (2015) A Triazine di(carboxy)porphyrin Dyad versus a Triazine di(carboxy)porphyrin Triad for Sensitizers in DSSCs. Dalton Transactions, 44, 13550-13564. https://doi.org/10.1039/C5DT01141H

[22] Zegkinoglou, I., Ragoussi, M.-E., Pemmaraju, C.D., Johnson, P.S., Pickup, D.F., Ortega, J.E., Prendergast, D., Torre, G. and Himpsel, F.J. (2013) Spectroscopy of Donor- $\pi$-Acceptor Porphyrins for Dye-Sensitized Solar Cells. The Journal of Physical Chemistry C, 117, 13357-13364. https://doi.org/10.1021/jp402590u 
[23] Song, H., Kong, I., Ko, I., Kang, J., Kim, Y.J., Kweon, J.U., Cha, Y., Kim, E., Jeon, T.S. and Choi, E.-Y. (2016) A Study of the CO2/CO/CH4 Adsorption Ability of Porphyrin Paddle-Wheel Frameworks (PPFs). Polymer Bulletin, 73, 2593-2603. https://doi.org/10.1007/s00289-016-1690-7

[24] Neti, V.S.P.K., Wu, X., Deng, S. and Echegoyen, L. (2013) Selective CO2 Capture in an Imine Linked Porphyrin Porous Polymer. Polymer Chemistry, 4, 4566-4569. https://doi.org/10.1039/c3py00798g

[25] Hod, I., Sampson, M.D., Deria, P., Kubiak, C.P., Farha, O.K. and Hupp, J.T. (2015) Fe-Porphyrin-Based Metal-Organic Framework Films as High-Surface Concentration, Heterogeneous Catalysts for Electrochemical Reduction of CO2. ACS Catalysis, 5, 6302-6309. https://doi.org/10.1021/acscatal.5b01767

[26] Kornienko, N., Zhao, Y., Kley, C.S., Zhu, C., Kim, D., Lin, S., Chang, C.J., Yaghi, O.M. and Yang, P. (2015) Metal-Organic Frameworks for Electrocatalytic Reduction of Carbon Dioxide. Journal of the American Chemical Society, 137, 14129-14135. https://doi.org/10.1021/jacs.5b08212

[27] Feng, D., Chung, W.-C., Wei, Z., Gu, Z.-Y., Jiang, H.-L., Chen, Y.-P., Darensbourg, D.J. and Zhou, H.-C. (2013) Construction of Ultrastable Porphyrin Zr Metal-Organic Frameworks through Linker Elimination. Journal of the American Chemical Society, 135, 17105-17110. https://doi.org/10.1021/ja408084j

[28] Feng, D., Gu, Z.-Y., Li, J.-R., Jiang, H.-L., Wei, Z. and Zhou, H.-C. (2012) Zirconium-Metalloporphyrin PCN-222: Mesoporous Metal-Organic Frameworks with Ultrahigh Stability as Biomimetic Catalysts. Angewandte Chemie International Edition, 51, 10307-10310. https://doi.org/10.1002/anie.201204475

[29] Wang, X.-S., Chrzanowski, M., Yuan, D., Sweeting, B.S. and Ma, S. (2014) Covalent Heme Framework as a Highly Active Heterogeneous Biomimetic Oxidation Catalyst. Chemistry of Materials, 26, 1639-1644. https://doi.org/10.1021/cm403860t

[30] Yao, B., Li, C., Ma, J. and Shi, G. (2015) Porphyrin-Based Graphene Oxide Frameworks with Ultra-Large d-Spacings for the Electrocatalyzation of Oxygen Reduction Reaction. Physical Chemistry Chemical Physics, 17, 19538-19545. https://doi.org/10.1039/C5CP02853A

[31] Ma, W., Yu, P., Ohsaka, T. and Mao, L. (2015) An Efficient Electrocatalyst for Oxygen Reduction Reaction Derived from a Co-Porphyrin-Based Covalent Organic Framework. Electrochemistry Communications, 52, 53-57. https://doi.org/10.1016/j.elecom.2015.01.021

[32] Xie, M.-H., Yang, X.-L., Zou, C. and Wu, C.-D. (2011) A SnIV Porphyrin-Based Metal Organic Framework for the Selective Photo-Oxygenation of Phenol and Sulfides. Inorganic Chemistry, 50, 5318-5320. https://doi.org/10.1021/ic200295h

[33] Fateeva, A., Chater, P.A., Ireland, C.P., Tahir, A.A., Khimyak, Y.Z., Wiper, P.V., Darwent, J.R. and Rosseinsky, M.J. (2012) A Water-Stable Porphyrin-Based Metal-Organic Framework Active for Visible-Light Photocatalysis. Angewandte Chemie International Edition, 51, 7440-7444. https://doi.org/10.1002/anie.201202471

[34] Johnson, J.A., Luo, J., Zhang, X., Chen, Y.-S., Morton, M.D., Echeverría, E., Torres, F.E. and Zhang, J. (2015) Porphyrin-Metalation-Mediated Tuning of Photoredox Catalytic Properties in Metal-Organic Frameworks. ACS Catalysis, 5, 5283-5291. https://doi.org/10.1021/acscatal.5b00941

[35] Ma, H., Ren, H., Meng, S., Sun, F. and Zhu, G. (2013) Novel Porphyrinic Porous Organic Frameworks for High Performance Separation of Small Hydrocarbons. Scientific Reports, 3, 2611. https://doi.org/10.1038/srep02611

[36] Pereira, C.F., Simoes, M.M.Q., Tome, J.P.C. and Paz, F.A.A. (2016) Porphy- 
rin-Based Metal-Organic Frameworks as Heterogeneous Catalysts in Oxidation Reactions. Molecules, 21, 1348. https://doi.org/10.3390/molecules21101348

[37] Goldberg, I. (2005) Crystal Engineering of Porphyrin Framework Solids. Chemical Communications, 2005, 1243-1254. https://doi.org/10.1039/b416425c

[38] Kandambeth, S., Shinde, D.B., Panda, M.K., Lukose, B., Heine, T. and Banerjeea, R. (2013) Enhancement of Chemical Stability and Crystallinity in Porphyrin Containing Covalent Organic Frameworks using Intra-Molecular Hydrogen Bonding. Angewandte Chemie, 52, 13052-13056. https://doi.org/10.1002/anie.201306775

[39] Zheng, G.D., Yan, Y., Gao, S., Tong, S.L. and Zhen, K.J. (1996) The Reaction Mechanism of Alkyl Halides with Carbon Dioxide Catalyzed by 5, 10, 15, 20-tetraphenyl Porphyrin Cobalt (CoTPP). Electrochemica Acta, 41, 177-182. https://doi.org/10.1016/0013-4686(95)00279-N

[40] Yan, Y., Xiao, F.-S., Zheng, G., Zhen, K. and Fang, C. (2000) Selective Catalytic Oxidation of Naphthol to 2-hydroxyl-1,4-naphthoquinone by Hydrogen Peroxide over Metalloporphyrin Catalysts. Journal of Molecular Catalysis A: Chemical, 157, 65-72. https://doi.org/10.1016/S1381-1169(99)00440-9

[41] Yan, Y., Kang, E.-H., Yang, K.-E., Tong, S.-L., Fang, C.-G., Liu, S.-J. and Xiao, F.-S. (2004) High Activity in Selective Catalytic Oxidation of Naphthol to 2-hydroxy-1,4-naphthoquinone by Molecular Oxygen under Air Pressure over Recycled Iron Porphyrin Catalysts. Catalysis Communications, 5, 387-390. https://doi.org/10.1016/j.catcom.2004.05.003 